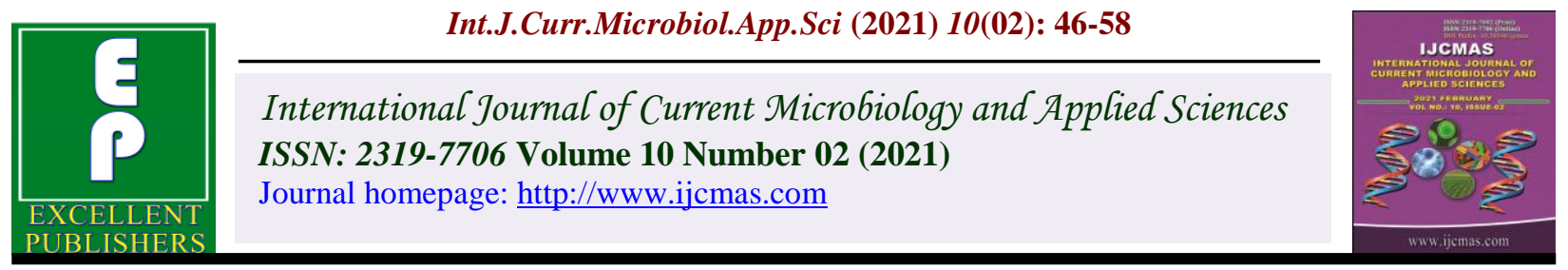

Original Research Article

https://doi.org/10.20546/ijcmas.2021.1002.006

\title{
Effect of Seed Polymerization with Micronutrients on Seed Yield and Quality in Biofortified Genotypes of Pearl Millet [Pennisetum glaucum (L.)]
}

\author{
S. Gireesh*, S. B. Patil, Ashok S. Sajjan and M. D. Patil \\ Department of Seed Science and Technology, College of Agriculture, Vijayapura, University \\ of Agricultural Sciences, Dharwad, (Karnataka), India \\ *Corresponding author
}

\section{A B S T R A C T}

Keywords

Pearlmillet, Polymerization, Micronutrients, Seed yield, Seed quality

Article Info

Accepted:

10 January 2021

Available Online:

10 February 2021
The field cum laboratory experiment was carried out in the Department of Seed Science and Technology, College of Agriculture, Vijayapur, University of Agricultural Sciences, Dharwad, during Kharif season of 2018-19 with an objective to study the effect of seed polymerization with micronutrients on seed yield and quality in biofortified genotypes of pearl millet (Pennisetum glaucum L.). The experiment was laid out in a factorial randomized block design with thirteen biofortified genotypes and twelve treatments including control with four replications. The results revealed that seeds treated with combined application of $5 \mathrm{ml}$ polymer per $\mathrm{kg}$ seed along with micronutrients namely, $\mathrm{ZnSo}_{4}+\mathrm{FeSo}_{4}+$ Boron + each at $2 \mathrm{~g}$ per $\mathrm{kg}$ of seed was recorded higher the seed yield and better quality in pearlmillet.

\section{Introduction}

Nutritional security is the key to improve the health status of the world's population as mankind is primarily dependent on plantbased diets. Plants are the major source of nutrients essential for normal growth and development. However, half of the global population, especially people from Asia and Africa suffer from nutrition deficiency as they rely on cereal crops for food (Hirschi, 2009). Pearl millet is a good source of energy, carbohydrate, protein, fat, ash, dietery fiber, iron and zinc. Pearl millet is a rich source of energy (361 Kcal/100g), The carbohydrate content of pearl millet is $67.5 \mathrm{~g}$ per $100 \mathrm{~g}$, with 56 to 65 percent starch comprising 20 to 22 percent amylase and 2.6 to 2.8 per cent free sugars.

Pearl millet is rich in fat content $(5 \mathrm{mg} / 100 \mathrm{~g})$ with better fat digestibility. It is rich in unsaturated fatty acids (75\%) with higher content of nutrionally important n-3 fatty acids. It is rich source of vitamins and minerals with overall minerals with overall mineral content of $2.3 \mathrm{mg}$ per $100 \mathrm{~g}$ constituting potassium, phosphorous, 
magnesium, iron, zinc, copper and manganese. It is rich in B-vitamins (thiamine, riboflavin and niacin).

Pearl millet (Pennisetum glaucum (L.) R. Br.) perform well under harsh environmental conditions with the annual rainfall about less than $250 \mathrm{~mm}$ and the temperature required is about $30{ }^{\circ} \mathrm{C}$ in loamy soils (Anon., 1996). It covers about 31 million hectares in the world. India is a competent pearl millet producer and it is grown over an area of 7.48 million hectares with a production of 9.21 million tonnes and mean productivity of $1231 \mathrm{~kg} \mathrm{ha}^{-1}$ during 2017-18 (Anonymous, 2020). Rajasthan, Haryana, Gujarat, Uttar Pradesh and Maharashtra are the key pearl millet cultivating states accounting beyond $90 \%$ of the India's pearl millet area. In India pearl millet is grown predominantly in kharif season. It is also grown during the summer season in certain regions of Uttar Pradesh, Gujarat and Rajasthan; and during the rabi season at a little extent in Gujarat and Maharashtra. In Karnataka, pearl millet is one of the major kharif crops, grown over an area of 2.42 lakh hectares with an annual production of 2.55 lakh tonnes with a mean productivity of $1054 \mathrm{~kg} \mathrm{ha}^{-1}$ (Anonymous, 2020).

Seed polymer is used for coating as a thin, uniform layer over the seeds without any significant increase in seed size or weight. The major benefits of using this seed polymer is that the seed enhancement materials (fungicides, microbiological agents and micronutrients) can be directly placed on to the seed which requires smaller amount of chemicals as compared to broadcasting or surface dressing. Seed coating is one of the most economical approaches for improving seed performance besides it has provoked the interest among many seed traders and seed companies, as it improves its marketability, brand identity and helps the farmers for easy identification of the crops and varieties based on colour. Modern seed technology provides a wide selection of enhancements that can be aimed at translating a variety's genetic potential into improved harvest and quality (Bharathi. A and Srinivasan. J. 2010).

The micronutrients may be supplied to the plants through soil application, foliar spray or applied to seed through seed treatment. Although the required amount of micronutrients can be supplied by any of these methods, foliar sprays have been more effective in yield improvement and grain enrichment. Application of micronutrients through foliar spray is taken up at later growth stages when crop stands are already established. Hence, an alternative acceptable method of supplying micronutrients during the early stage of seedling establishment is the need of the hour. Micronutrients often act as cofactors in enzyme systems and participate in redox reactions, in addition to having several other vital functions in plants. Most importantly, micronutrients are involved in the key physiological processes of photosynthesis and respiration and their deficiency can impede these vital physiological processes thus limiting yield gain in many crops. Owing to this, the productivity of many crops has reduced substantially over the years. It is estimated that, the extent of micronutrient deficiency in the Indian soils are 47, 35, 15, 13, 4 and 2 per cent for Zinc, Boron, Molybdenum, Iron, Manganese and Copper, respectively. While, in Karnataka it is 78, 39, 32 and 5 per cent for Zinc, Iron, Boron and Copper respectively (Anon., 2011).

Among the micronutrients zinc, ferrous sulphate and boron are very important for pearlmillet. Zinc is involved in biosynthesis of plant hormone, indole acetic acid (IAA), auxin metabolism and is component of variety of enzymes which plays an important role in 
nucleic acid and protein synthesis and helps in utilization of phosphorus and nitrogen in seed formation and development. The second phase of seed development requires adequate amount of zinc dependent upon auxin for seed development. Ferrous sulphate is an important constituent of chlorophyll biosynthesis, regulates respiration, photosynthesis, reduction of nitrates and sulphates, also activates several enzymes involved in respiration (Kaleeswari et al., 2013). While, boron plays an important role in flower retention, pollen tube growth, seed formation and seed setting and mainly involved in translocation of metabolites from source to sink (Dell and Huang, 1997; Tanaka and Fujiwar, 2008).

Micronutrient application through seed treatment improves the stand establishment, advances phenological events, increases yield and micronutrient contents in grain in most of the crops. In many cases, micronutrient application through seed treatment performed better or similar to other application methods (Singh et al., 2003). Being an easy and cost effective method, micronutrient application through seed treatment by polymer coating offer an attractive option for resource-poor farmers (Johnson et al., 2005).

Keeping in view of the above aspects, the present investigation was taken up in biofortified genotypes of pearlmillet on the effect of seed polymerization with micronutrients on seed yield and quality in biofortified genotypes of pearl millet [pennisetum glaucum (L.)].

\section{Materials and Methods}

The Field cum Laboratory experiment was conducted in the Department of Seed Science and Technology, College of Agriculture, Vijayapur. University of Agricultural Sciences, Dharwad. The experiment consisted of thirteen biofortified lines and twelve different treatments (including control) with four replications. The treatments were $\mathrm{T}_{0}$ : Control, $\mathrm{T}_{1}: \mathrm{ZnSO}_{4} @ 2 \mathrm{~g}$ per kg of seed, $\mathrm{T}_{2}$ : $\mathrm{ZnSO}_{4} @ 4 \mathrm{~g}$ per kg of seed, $\mathrm{T}_{3}: \mathrm{FeSO}_{4} @ 2 \mathrm{~g}$ per kg of seed, $\mathrm{T}_{4}: \mathrm{FeSO}_{4} @ 4 \mathrm{~g}$ per kg of seed, $\mathrm{T}_{5}$ : Boron @ $2 \mathrm{~g}$ per $\mathrm{kg}$ of seed, $\mathrm{T}_{6}$ : Boron@4g per kg of seed, $\mathrm{T}_{7}: \mathrm{ZnSO}_{4}+$ $\mathrm{FeSO}_{4}$ (each@2g/kg of seed), $\mathrm{T}_{8}: \mathrm{ZnSO}_{4}+$ $\mathrm{FeSO}_{4}$ (each@ @g/kg of seed), $\mathrm{T}_{9}: \mathrm{ZnSO}_{4}+$ $\mathrm{FeSO}_{4}+$ Boron (each @ $2 \mathrm{~g} / \mathrm{kg}$ of seed), $\mathrm{T}_{10}$ : $\mathrm{ZnSO}_{4}+\mathrm{FeSO}_{4}+$ Boron (each @ 4g/kg of seed), $\mathrm{T}_{11}$ : Only polymer. The genotypes were $\mathrm{G}_{1}$ : Fe \& Zn 2019-2R, G $\mathrm{G}_{3}$ : Fe \& Zn 2019-6R, G $\mathrm{G}_{5}$ : Fe \& Zn 2019-10R, G6 : Fe \& Zn 201912R, G $:$ Fe \& Zn 2019-16R, G8: Fe \& Zn 2019-21R, G9: Fe \& Zn 2019-22R, G 10 : Fe \& Zn 2019-26R, G ${ }_{11}$ : DHANASHAKTHI, G ${ }_{12}$ : ICTP 8203, G 13 :VPMH-7.

The experiment was laid in factorial completely randomized design for seed of different treatment combination treated with polymer along with micronutrients. The standardized dosage of polymer coating of 5 $\mathrm{ml}$ per $\mathrm{kg}$ of seed was used. The crop was raised by adopting recommended fertilizer dose of 100:65:25 NPK kg/ha was applied as basal dose at the time of sowing, sowing of polymer coated seeds of pearl millet was done as per the treatment details with two seeds per hill by hand dibbling on one side of the ridge at a spacing of $45 \times 15 \mathrm{~cm}$. Soon after sowing, the experimental plots were irrigated lightly to ensure uniform germination and optimum plant stand. The observations were recorded on seed yield and quality parameters.

\section{Procedure for polymer coating of seeds}

The polymer used in the present study was Disco Agro DC Red L-603 procured from Incotec Pvt. Ltd. Ahmedabad, Gujarat. The cleaned and graded seeds were coated with 
different micronutrients as per the treatments either individually or in combination with polymer @ $5 \mathrm{ml}$ per kg of seed using rotary seed coating machine. Subsequently the seeds were air dried to bring back to original moisture content and then used for seed quality testing.

The Seed germination test was conducted as per ISTA (Anon., 2014), shoot length, root length, seedling dry weight as per Evans and Bhatt (1977), seedling vigour index was worked out as per the formula given by Abdul-Baki and Anderson (1973). The data of the laboratory experiment were analyzed statistically by the procedure prescribed by Gomez and Gomez (2010).

\section{Results and Discussion}

The seed yield differed significantly due to seed polymerization with a micronutrients. The higher seed yield of 24.65 per cent over control recorded due to seed polymerized with $\mathrm{ZnSO}_{4}+\mathrm{FeSO}_{4}+$ boron each @ 2g per $\mathrm{kg}$ (Table 1). The maximum seed yield (2520 $\mathrm{kg} / \mathrm{ha}$ ) noticed in seed polymerized with $\mathrm{ZnSO}_{4}+\mathrm{FeSO}_{4}+$ boron each @ $2 \mathrm{~g}$ per kg of seed, followed by $\mathrm{ZnSO}_{4}+\mathrm{FeSO}_{4}+$ boron each @ 4g per kg of seed (2393 kg/ha), and seed polymerized with $\mathrm{ZnSO}_{4}+\mathrm{FeSO}_{4}$ each (a) $2 \mathrm{~g}$ per $\mathrm{kg}$ of seed $(2345 \mathrm{~kg} / \mathrm{ha})$. While, the lowest seed yield was seen in control (1898.32 kg/ha).

Increase in yield attributing characters due to seed treatment with micronutrients and these might be due to accumulation of photo as similates and partitioning in different parts of the plant. The yield is strongly influenced by the application of micronutrients, that indicating the role of these micronutrients in increasing the yield through their effect on various morpho- physiological traits. These results were in conformity with Sharma et al., (2017), Shinde et al., (2018).
Seed quality parameters were influenced significantly due to the seed polymerization with a micronutrients treatments(Table 2 to 6). The higher Seedling Vigour index - I (3452) and Seedling Vigour index - II (7796) observed due to seed polymerized with $\mathrm{ZnSO}_{4}+\mathrm{FeSO}_{4}+$ boron each @ $2 \mathrm{~g}$ per kg of seed.

The increased Seedling Vigour index - I and Seedling Vigour index - II mainly attributed by higher Germination percentage, seedling length and Seedling dry weight were recorded in seed polymerized with $\mathrm{ZnSO}_{4}+\mathrm{FeSO}_{4}+$ boron each@2g per kg. (94.65\%, 36.80 cm and $82.28 \mathrm{~g}$ respectively) followed by seed polymerized with $\mathrm{ZnSO}_{4}+\mathrm{FeSO}_{4}+$ boron each @ 4g per kg $(93.34 \%, 34.78 \mathrm{~cm}$ and $80.43 \mathrm{~g}$, respectively) and seed polymerized with $\mathrm{ZnSO}_{4}+\mathrm{FeSO}_{4}$ each @ 2g per kg of seed $(92.31 \%, 35.61 \mathrm{~cm}$ and $79.52 \mathrm{~g}$ respectively).

While, the lower seed quality parameters was recorded in control $(85.56 \%, 30.65 \mathrm{~cm}$ and $70.88 \mathrm{~g}$ respectively). This is having a positive relation to seed development which is reflected through the size and test weight of seed and the micronutrients present in it.

This in turn dependant on efficient synthesis, accumulation of food metabolites such as protein, carbohydrates and their translocation from source to the developing seed at greater ease. Seed with higher initial capital food reserves always showed higher and rapid germination which is also true in present study.

Similarly, the micronutrients also participate in catalytic activity and breakdown of complex food source to simple form (glucose, amino acids and fatty acids). These in turn reflected on enhancing the rate of germination and elongation of root and shoot. 
Table.1 Effect of seed polymerization with micronutrients on seed yield $(\mathrm{kg} / \mathrm{ha})$ in different biofortified genotypes of pearl millet

\begin{tabular}{|c|c|c|c|c|c|c|c|c|c|c|c|c|c|}
\hline $\mathbf{G} / \mathbf{T}$ & $\mathbf{T}_{\mathbf{0}}$ & $\mathbf{T}_{1}$ & $\mathbf{T}_{2}$ & $\mathbf{T}_{3}$ & $\mathbf{T}_{4}$ & $\mathbf{T}_{5}$ & $\mathbf{T}_{6}$ & $\mathbf{T}_{7}$ & $\mathbf{T}_{8}$ & $\mathbf{T}_{9}$ & $\mathbf{T}_{10}$ & T11 & Mean \\
\hline $\mathbf{G}_{1}$ & 2218 & 2079 & 2141 & 2105 & 1722 & 1902 & 2054 & 2252 & 2120 & 2317 & 2282 & 1703 & 2075 \\
\hline $\mathbf{G}_{2}$ & 1668 & 1916 & 1776 & 1891 & 1820 & 1971 & 1699 & 2065 & 2215 & 2195 & 2254 & 1724 & 1933 \\
\hline $\mathbf{G}_{3}$ & 1756 & 2075 & 2156 & 1893 & 2045 & 2017 & 2120 & 2349 & 2162 & 2369 & 2279 & 2021 & 2103 \\
\hline $\mathbf{G}_{4}$ & 1842 & 2305 & 2043 & 2121 & 1949 & 2113 & 1971 & 1854 & 2063 & 2480 & 2317 & 1847 & 2076 \\
\hline $\mathbf{G}_{5}$ & 1824 & 1873 & 1849 & 1843 & 1849 & 1794 & 1809 & 2372 & 2143 & 2579 & 1866 & 2305 & 2009 \\
\hline $\mathbf{G}_{6}$ & 1778 & 2097 & 2178 & 1916 & 2067 & 2039 & 2142 & 2372 & 2184 & 2391 & 2301 & 2043 & 2126 \\
\hline $\mathbf{G}_{7}$ & 1990 & 2592 & 2471 & 2492 & 2495 & 2369 & 2579 & 2454 & 2559 & 2813 & 2782 & 2606 & 2517 \\
\hline $\mathbf{G}_{8}$ & 1758 & 2114 & 2117 & 1940 & 1992 & 1866 & 1944 & 2192 & 1973 & 2441 & 2233 & 2191 & 2063 \\
\hline $\mathbf{G}_{9}$ & 1701 & 1703 & 2070 & 1823 & 2005 & 1743 & 1828 & 2279 & 2153 & 2395 & 2252 & 2043 & 2000 \\
\hline $\mathbf{G}_{10}$ & 2113 & 2481 & 2567 & 2566 & 2780 & 2658 & 2504 & 2775 & 2633 & 2819 & 2805 & 2606 & 2609 \\
\hline G11 & 1971 & 2225 & 2424 & 2172 & 2410 & 2152 & 2425 & 2405 & 2394 & 2466 & 2388 & 1737 & 2264 \\
\hline G12 & 1990 & 2435 & 2408 & 2417 & 2465 & 2497 & 2420 & 2356 & 2423 & 2637 & 2536 & 2278 & 2405 \\
\hline G13 & 2068 & 2471 & 2772 & 2718 & 2765 & 2716 & 2780 & 2754 & 2764 & 2855 & 2811 & 2533 & 2667 \\
\hline Mean & 1898 & 2182 & 2229 & 2146 & 2182 & 2141 & 2175 & 2345 & 2291 & 2520 & 2393 & 2126 & \\
\hline $\begin{array}{c}\text { For } \\
\text { comparing } \\
\text { the means of }\end{array}$ & $\begin{array}{c}\text { S.Em } \\
\pm\end{array}$ & $\begin{array}{c}\text { CD at } 5 \\
\%\end{array}$ & & & & & & & & & & & \\
\hline $\mathbf{T}$ & 36.71 & 102.14 & & & & & & & & & & & \\
\hline G & 38.21 & 106.32 & & & & & & & & & & & \\
\hline T X G & 132.35 & NS & & & & & & & & & & & \\
\hline
\end{tabular}

Note: NS-Non significant

LEGEND: Treatments (T) $\mathrm{T}_{0}$ : Control, $\mathrm{T}_{1}: \mathrm{ZnSO}_{4} @ 2 \mathrm{~g}$ per kg of seed, $\mathrm{T}_{2}: \mathrm{ZnSO}_{4} @ 4 \mathrm{~g}$ per kg of seed, $\mathrm{T}_{3}: \mathrm{FeSO}_{4} @ 2 \mathrm{~g} \mathrm{per} \mathrm{kg} \mathrm{of} \mathrm{seed,} \mathrm{T}_{4}: \mathrm{FeSO}_{4}$ @ $4 \mathrm{~g}$ per kg of seed, $\mathrm{T}_{5}$ : Boron@ $2 \mathrm{~g}$ per kg of seed, $\mathrm{T}_{6}$ : Boron @ 4g per kg of seed, $\mathrm{T}_{7}: \mathrm{ZnSO}_{4}+\mathrm{FeSO}_{4}\left(\mathrm{each} @ 2 \mathrm{~g} / \mathrm{kg}\right.$ of seed), $\mathrm{T}_{8}: \mathrm{ZnSO} 4+$ $\mathrm{FeSO}_{4}$ (each@ @ $\mathrm{g} / \mathrm{kg}$ of seed), $\mathrm{T}_{9}: \mathrm{ZnSO}_{4}+\mathrm{FeSO}_{4}+$ Boron (each @ 2g/ kg of seed), $\mathrm{T}_{10}: \mathrm{ZnSO}_{4}+\mathrm{FeSO}_{4}+\mathrm{Boron}\left(\mathrm{each} @ 4 \mathrm{~g} / \mathrm{kg}\right.$ of seed), $\mathrm{T}_{11}$ : Onlypolymer

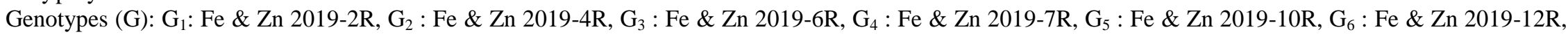
$\mathrm{G}_{7}$ : Fe \& Zn 2019-16R, G 8 : Fe \& Zn 2019-21R, G $:$ Fe \& Zn 2019-22R, G 10 : Fe \& Zn 2019-26R, G 1 : DHANASHAKTHI, G 12 : ICTP 8203, G 13 : VPMH-7 
Table.2 Effect of seed polymerization with micronutrients on germination percentage of resultant seeds of biofortified genotypes of pearl millet

\begin{tabular}{|c|c|c|c|c|c|c|c|c|c|c|c|c|c|}
\hline G/T & $\mathbf{T}_{\mathbf{0}}$ & $\mathbf{T}_{1}$ & $\begin{array}{l}\mathbf{T} \\
2\end{array}$ & $\mathbf{T}_{3}$ & $\begin{array}{l}\mathbf{T} \\
4\end{array}$ & $\begin{array}{l}\mathbf{T} \\
5\end{array}$ & $\begin{array}{l}T \\
6 \\
\end{array}$ & $\mathbf{T}_{7}$ & $\begin{array}{l}\mathrm{T} \\
8\end{array}$ & $\mathbf{T}_{9}$ & $\mathbf{T}_{10}$ & $\mathbf{T}_{11}$ & Mean \\
\hline $\mathbf{G}_{1}$ & $\begin{array}{c}84.27 \\
(66.63)\end{array}$ & $\begin{array}{c}84.43 \\
(66.76)\end{array}$ & $\begin{array}{c}85.43 \\
(67.56)\end{array}$ & $\begin{array}{c}84.33 \\
(66.68) \\
\end{array}$ & $\begin{array}{c}84.03 \\
(66.45)\end{array}$ & $\begin{array}{c}84.43 \\
(66.76) \\
\end{array}$ & $\begin{array}{c}88.47 \\
(70.15)\end{array}$ & $\begin{array}{c}93.97 \\
(75.59)\end{array}$ & $\begin{array}{c}89.43 \\
(71.03)\end{array}$ & $\begin{array}{c}94.43 \\
(76.35)\end{array}$ & $\begin{array}{c}91.43 \\
(72.98)\end{array}$ & $\begin{array}{c}84.93 \\
(67.16)\end{array}$ & $\begin{array}{r}87.47 \\
(69.52)\end{array}$ \\
\hline $\mathbf{G}_{2}$ & $\begin{array}{c}84.13 \\
(66.52)\end{array}$ & $\begin{array}{c}84.67 \\
(66.95)\end{array}$ & $\begin{array}{c}85.33 \\
(67.48)\end{array}$ & $\begin{array}{c}84.33 \\
(66.68)\end{array}$ & $\begin{array}{c}89.33 \\
(70.93)\end{array}$ & $\begin{array}{c}86.67 \\
(68.59) \\
\end{array}$ & $\begin{array}{c}87.70 \\
(69.47)\end{array}$ & $\begin{array}{c}85.83 \\
(67.89)\end{array}$ & $\begin{array}{c}86.33 \\
(68.30)\end{array}$ & $\begin{array}{c}86.92 \\
(68.80) \\
\end{array}$ & $\begin{array}{c}90.33 \\
(71.88)\end{array}$ & $\begin{array}{c}88.83 \\
(70.48)\end{array}$ & $\begin{array}{r}86.70 \\
(68.66)\end{array}$ \\
\hline $\mathbf{G}_{3}$ & $\begin{array}{c}82.83 \\
(65.52)\end{array}$ & $\begin{array}{c}85.33 \\
(67.48)\end{array}$ & $\begin{array}{c}85.33 \\
(67.48)\end{array}$ & $\begin{array}{c}94.33 \\
(76.22)\end{array}$ & $\begin{array}{c}85.33 \\
(67.48)\end{array}$ & $\begin{array}{c}84.33 \\
(66.68)\end{array}$ & $\begin{array}{c}87.03 \\
(68.89)\end{array}$ & $\begin{array}{c}92.20 \\
(73.78)\end{array}$ & $\begin{array}{c}91.33 \\
(72.88)\end{array}$ & $\begin{array}{c}93.57 \\
(75.31)\end{array}$ & $\begin{array}{c}93.67 \\
(75.43)\end{array}$ & $\begin{array}{c}84.83 \\
(67.08)\end{array}$ & $\begin{array}{r}88.34 \\
(70.35)\end{array}$ \\
\hline $\mathbf{G}_{4}$ & $\begin{array}{c}87.83 \\
(69.58)\end{array}$ & $\begin{array}{c}85.33 \\
(67.48)\end{array}$ & $\begin{array}{c}91.33 \\
(72.88)\end{array}$ & $\begin{array}{c}84.80 \\
(67.05)\end{array}$ & $\begin{array}{c}89.33 \\
(70.93)\end{array}$ & $\begin{array}{c}86.67 \\
(68.59)\end{array}$ & $\begin{array}{c}91.03 \\
(72.57)\end{array}$ & $\begin{array}{c}93.20 \\
(74.88)\end{array}$ & $\begin{array}{c}91.33 \\
(72.88)\end{array}$ & $\begin{array}{c}95.67 \\
(77.99)\end{array}$ & $\begin{array}{c}90.00 \\
(71.57)\end{array}$ & $\begin{array}{c}85.83 \\
(67.89)\end{array}$ & $\begin{array}{r}89.36 \\
(71.19)\end{array}$ \\
\hline $\mathbf{G}_{5}$ & $\begin{array}{c}85.33 \\
(67.48)\end{array}$ & $\begin{array}{c}93.83 \\
(75.62)\end{array}$ & $\begin{array}{c}86.83 \\
(68.72)\end{array}$ & $\begin{array}{c}83.83 \\
(66.29)\end{array}$ & $\begin{array}{c}87.83 \\
(69.58)\end{array}$ & $\begin{array}{c}94.83 \\
(76.86)\end{array}$ & $\begin{array}{c}84.53 \\
(66.84)\end{array}$ & $\begin{array}{c}88.70 \\
(70.36)\end{array}$ & $\begin{array}{c}90.83 \\
(72.37)\end{array}$ & $\begin{array}{c}94.50 \\
(76.44)\end{array}$ & $\begin{array}{c}92.17 \\
(73.75)\end{array}$ & $\begin{array}{c}87.33 \\
(69.15)\end{array}$ & $\begin{array}{r}89.21 \\
(71.12)\end{array}$ \\
\hline $\mathbf{G}_{6}$ & $\begin{array}{c}84.17 \\
(66.56)\end{array}$ & $\begin{array}{c}85.33 \\
(67.48)\end{array}$ & $\begin{array}{c}84.67 \\
(66.95)\end{array}$ & $\begin{array}{c}86.33 \\
(68.30)\end{array}$ & $\begin{array}{c}83.83 \\
(66.29)\end{array}$ & $\begin{array}{c}84.33 \\
(66.68)\end{array}$ & $\begin{array}{c}84.03 \\
(66.45)\end{array}$ & $\begin{array}{c}93.53 \\
(75.26)\end{array}$ & $\begin{array}{c}88.33 \\
(70.02)\end{array}$ & $\begin{array}{c}95.67 \\
(77.99)\end{array}$ & $\begin{array}{c}90.67 \\
(72.21)\end{array}$ & $\begin{array}{c}84.83 \\
(67.08)\end{array}$ & $\begin{array}{r}87.14 \\
(69.27)\end{array}$ \\
\hline $\mathbf{G}_{7}$ & $\begin{array}{c}86.66 \\
(68.58)\end{array}$ & $\begin{array}{c}84.93 \\
(67.16)\end{array}$ & $\begin{array}{c}92.83 \\
(74.47)\end{array}$ & $\begin{array}{c}85.73 \\
(67.81)\end{array}$ & $\begin{array}{c}95.50 \\
(77.75)\end{array}$ & $\begin{array}{c}88.23 \\
(69.94)\end{array}$ & $\begin{array}{c}90.87 \\
(72.41)\end{array}$ & $\begin{array}{c}94.93 \\
(76.99)\end{array}$ & $\begin{array}{c}95.33 \\
(77.52)\end{array}$ & $\begin{array}{c}96.47 \\
(79.17)\end{array}$ & $\begin{array}{c}95.50 \\
(77.75)\end{array}$ & $\begin{array}{c}93.33 \\
(75.03)\end{array}$ & $\begin{array}{c}91.69 \\
(73.71)\end{array}$ \\
\hline $\mathbf{G}_{8}$ & $\begin{array}{c}87.50 \\
(69.30)\end{array}$ & $\begin{array}{c}84.33 \\
(66.68)\end{array}$ & $\begin{array}{c}89.33 \\
(70.93)\end{array}$ & $\begin{array}{c}87.33 \\
(69.15)\end{array}$ & $\begin{array}{c}86.33 \\
(68.30)\end{array}$ & $\begin{array}{c}78.33 \\
(62.26)\end{array}$ & $\begin{array}{c}94.70 \\
(76.69)\end{array}$ & $\begin{array}{c}95.53 \\
(77.79)\end{array}$ & $\begin{array}{c}88.33 \\
(70.02)\end{array}$ & $\begin{array}{c}95.87 \\
(78.27)\end{array}$ & $\begin{array}{c}95.31 \\
(77.49)\end{array}$ & $\begin{array}{c}85.63 \\
(67.72)\end{array}$ & $\begin{array}{r}89.04 \\
(71.22)\end{array}$ \\
\hline $\mathbf{G}_{9}$ & $\begin{array}{c}84.83 \\
(67.08)\end{array}$ & $\begin{array}{c}95.33 \\
(77.52)\end{array}$ & $\begin{array}{c}86.33 \\
(68.30)\end{array}$ & $\begin{array}{c}85.33 \\
(67.48)\end{array}$ & $\begin{array}{c}91.33 \\
(72.88)\end{array}$ & $\begin{array}{c}85.67 \\
(67.76)\end{array}$ & $\begin{array}{c}89.33 \\
(70.93)\end{array}$ & $\begin{array}{c}86.53 \\
(68.47)\end{array}$ & $\begin{array}{c}89.33 \\
(70.93)\end{array}$ & $\begin{array}{c}93.57 \\
(75.31)\end{array}$ & $\begin{array}{c}94.33 \\
(76.22)\end{array}$ & $\begin{array}{c}84.83 \\
(67.08)\end{array}$ & $\begin{array}{r}88.90 \\
(70.83)\end{array}$ \\
\hline $\mathbf{G}_{10}$ & $\begin{array}{c}85.93 \\
(67.97)\end{array}$ & $\begin{array}{c}88.63 \\
(70.29)\end{array}$ & $\begin{array}{c}95.60 \\
(77.89)\end{array}$ & $\begin{array}{c}94.63 \\
(76.60)\end{array}$ & $\begin{array}{c}92.63 \\
(74.25)\end{array}$ & $\begin{array}{c}95.60 \\
(77.89)\end{array}$ & $\begin{array}{c}91.33 \\
(72.88)\end{array}$ & $\begin{array}{c}94.83 \\
(76.86)\end{array}$ & $\begin{array}{c}92.63 \\
(74.25)\end{array}$ & $\begin{array}{c}96.63 \\
(79.42)\end{array}$ & $\begin{array}{c}95.83 \\
(78.22)\end{array}$ & $\begin{array}{c}86.13 \\
(68.13)\end{array}$ & $\begin{array}{r}92.53 \\
(74.55)\end{array}$ \\
\hline $\mathbf{G}_{11}$ & $\begin{array}{c}86.33 \\
(68.30) \\
\end{array}$ & $\begin{array}{c}85.83 \\
(67.89) \\
\end{array}$ & $\begin{array}{c}90.83 \\
(72.37)\end{array}$ & $\begin{array}{c}88.83 \\
(70.48)\end{array}$ & $\begin{array}{c}91.83 \\
(73.39) \\
\end{array}$ & $\begin{array}{c}84.83 \\
(67.08) \\
\end{array}$ & $\begin{array}{c}93.53 \\
(75.26)\end{array}$ & $\begin{array}{c}91.03 \\
(72.57)\end{array}$ & $\begin{array}{c}94.73 \\
(76.73) \\
\end{array}$ & $\begin{array}{c}94.93 \\
(76.99) \\
\end{array}$ & $\begin{array}{c}94.83 \\
(76.86)\end{array}$ & $\begin{array}{c}85.33 \\
(67.48) \\
\end{array}$ & $\begin{array}{c}90.24 \\
(72.12)\end{array}$ \\
\hline $\mathbf{G}_{12}$ & $\begin{array}{c}87.65 \\
(69.43)\end{array}$ & $\begin{array}{c}91.33 \\
(72.88)\end{array}$ & $\begin{array}{c}95.13 \\
(77.25)\end{array}$ & $\begin{array}{c}87.13 \\
(68.98)\end{array}$ & $\begin{array}{c}85.47 \\
(67.59)\end{array}$ & $\begin{array}{c}90.13 \\
(71.69)\end{array}$ & $\begin{array}{c}85.83 \\
(67.89)\end{array}$ & $\begin{array}{c}94.33 \\
(76.22)\end{array}$ & $\begin{array}{c}95.13 \\
(77.25)\end{array}$ & $\begin{array}{c}95.13 \\
(77.25)\end{array}$ & $\begin{array}{c}93.47 \\
(75.19)\end{array}$ & $\begin{array}{c}89.63 \\
(71.21)\end{array}$ & $\begin{array}{r}90.86 \\
(72.74)\end{array}$ \\
\hline $\mathbf{G}_{13}$ & $\begin{array}{c}84.83 \\
(67.08)\end{array}$ & $\begin{array}{c}94.43 \\
(76.35)\end{array}$ & $\begin{array}{c}94.23 \\
(76.10)\end{array}$ & $\begin{array}{c}93.62 \\
(75.37)\end{array}$ & $\begin{array}{c}94.10 \\
(75.94)\end{array}$ & $\begin{array}{c}95.63 \\
(77.93)\end{array}$ & $\begin{array}{c}92.13 \\
(73.71)\end{array}$ & $\begin{array}{c}95.43 \\
(77.66)\end{array}$ & $\begin{array}{c}95.33 \\
(77.52)\end{array}$ & $\begin{array}{c}97.13 \\
(80.25)\end{array}$ & $\begin{array}{c}95.93 \\
(78.36)\end{array}$ & $\begin{array}{c}86.27 \\
(68.25)\end{array}$ & $\begin{array}{r}93.26 \\
(75.38)\end{array}$ \\
\hline Mean & $\begin{array}{c}85.56 \\
(67.69)\end{array}$ & $\begin{array}{c}87.98 \\
(70.04)\end{array}$ & $\begin{array}{c}89.48 \\
(71.41)\end{array}$ & $\begin{array}{c}87.73 \\
(69.78)\end{array}$ & $\begin{array}{c}88.99 \\
(70.91)\end{array}$ & $\begin{array}{c}87.67 \\
(69.90)\end{array}$ & $\begin{array}{c}89.27 \\
(71.09)\end{array}$ & $\begin{array}{c}92.31 \\
(741.19 \\
)\end{array}$ & $\begin{array}{c}91.41 \\
(73.21)\end{array}$ & $\begin{array}{c}94.65 \\
(76.89)\end{array}$ & $\begin{array}{c}93.34 \\
(75.22)\end{array}$ & $\begin{array}{c}86.75 \\
(68.75)\end{array}$ & \\
\hline $\begin{array}{l}\text { For comparing } \\
\text { the means of }\end{array}$ & $\begin{array}{l}\text { S.Em } \\
\pm\end{array}$ & CD at $1 \%$ & & & & & & & & & & & \\
\hline $\mathbf{T}$ & 0.65 & 2.40 & & & & & & & & & & & \\
\hline G & 0.68 & 2.50 & & & & & & & & & & & \\
\hline $\begin{array}{c}\text { T X } \\
\mathbf{G}\end{array}$ & 2.36 & NS & & & & & & & & & & & \\
\hline
\end{tabular}

*Figures in the parenthesis indicate arcsine transformed values, Note: NS-Non significant 
Table.3 Effect of seed polymerization with micronutrients on seedling length $(\mathrm{cm})$ of resultant seeds of biofortified genotypes of pearl millet

\begin{tabular}{|c|c|c|c|c|c|c|c|c|c|c|c|c|c|}
\hline $\mathbf{G} / \mathbf{T}$ & $\mathbf{T}_{\mathbf{0}}$ & $\mathrm{T}_{1}$ & $\mathbf{T}_{2}$ & $\mathbf{T}_{\mathbf{3}}$ & $\mathbf{T}_{4}$ & $\mathbf{T}_{5}$ & $\mathbf{T}_{6}$ & $\mathbf{T}_{7}$ & $\mathbf{T}_{8}$ & $\mathbf{T}_{9}$ & T10 & T11 & Mean \\
\hline $\mathbf{G}_{1}$ & 29.13 & 33.57 & 33.90 & 27.30 & 30.54 & 25.52 & 33.80 & 34.42 & 36.01 & 37.70 & 36.20 & 29.10 & 32.27 \\
\hline $\mathbf{G}_{2}$ & 28.10 & 34.83 & 33.89 & 28.20 & 31.54 & 26.10 & 25.60 & 34.90 & 36.98 & 34.80 & 37.09 & 27.70 & 31.64 \\
\hline $\mathbf{G}_{3}$ & 28.82 & 33.87 & 32.00 & 35.92 & 32.49 & 29.60 & 36.18 & 33.84 & 33.30 & 36.45 & 35.70 & 32.50 & 33.39 \\
\hline $\mathbf{G}_{4}$ & 32.12 & 34.76 & 34.60 & 28.57 & 31.19 & 31.51 & 31.30 & 35.70 & 34.39 & 35.90 & 28.10 & 31.53 & 32.47 \\
\hline $\mathbf{G}_{5}$ & 28.80 & 31.17 & 28.60 & 33.00 & 33.87 & 36.70 & 32.90 & 37.10 & 31.00 & 36.04 & 33.20 & 32.23 & 32.88 \\
\hline $\mathbf{G}_{6}$ & 27.50 & 31.75 & 31.40 & 31.63 & 32.27 & 28.70 & 31.00 & 34.30 & 31.00 & 37.70 & 32.60 & 33.36 & 31.93 \\
\hline $\mathbf{G}_{7}$ & 28.81 & 33.76 & 35.59 & 35.16 & 33.58 & 39.39 & 36.09 & 37.09 & 39.29 & 34.49 & 35.32 & 34.89 & 35.29 \\
\hline $\mathbf{G}_{8}$ & 32.79 & 28.78 & 32.90 & 33.03 & 33.99 & 27.78 & 27.30 & 35.50 & 30.60 & 38.38 & 33.20 & 34.64 & 32.41 \\
\hline $\mathbf{G}_{9}$ & 28.33 & 28.00 & 32.33 & 34.19 & 32.02 & 29.89 & 27.03 & 36.23 & 32.17 & 36.43 & 34.13 & 33.18 & 31.99 \\
\hline G10 & 29.80 & 35.07 & 36.95 & 35.90 & 33.69 & 40.00 & 36.20 & 37.30 & 39.30 & 35.00 & 36.10 & 35.75 & 35.92 \\
\hline G11 & 31.40 & 32.63 & 34.00 & 33.60 & 34.59 & 28.58 & 31.70 & 32.20 & 34.50 & 38.20 & 40.40 & 35.10 & 33.91 \\
\hline G12 & 37.52 & 30.47 & 33.50 & 30.44 & 32.73 & 33.19 & 32.80 & 36.31 & 37.72 & 38.10 & 33.10 & 39.10 & 34.58 \\
\hline $\mathbf{G}_{13}$ & 35.30 & 36.37 & 38.09 & 37.20 & 37.39 & 37.70 & 32.60 & 38.11 & 40.34 & 39.20 & 37.00 & 36.40 & 37.14 \\
\hline Mean & 30.65 & 32.69 & 33.67 & 32.63 & 33.07 & 31.90 & 31.89 & 35.61 & 35.12 & 36.80 & 34.78 & 33.50 & \\
\hline $\begin{array}{l}\text { For } \\
\text { comparing } \\
\text { mean of }\end{array}$ & S.Em \pm & $\begin{array}{c}\text { CD at } \\
1 \%\end{array}$ & & & & & & & & & & & \\
\hline $\mathbf{T}$ & 0.22 & 0.61 & & & & & & & & & & & \\
\hline G & 0.23 & 0.64 & & & & & & & & & & & \\
\hline T X G & 0.79 & NS & & & & & & & & & & & \\
\hline
\end{tabular}

Note: NS-Non significant

LEGEND: Treatments (T) $\mathrm{T}_{0}:$ Control, $\mathrm{T}_{1}: \mathrm{ZnSO}_{4} @ 2 \mathrm{~g}$ per kg of seed, $\mathrm{T}_{2}: \mathrm{ZnSO}_{4} @ 4 \mathrm{~g}$ per kg of seed, $\mathrm{T}_{3}: \mathrm{FeSO}_{4} @ 2 \mathrm{~g}$ per kg of seed, $\mathrm{T}_{4}:$ FeSO @ $4 \mathrm{~g}$ per kg of seed, $\mathrm{T}_{5}$ : Boron @ $2 \mathrm{~g}$ per kg of seed, $\mathrm{T}_{6}$ : Boron @ $4 \mathrm{~g}$ per kg of seed, $\mathrm{T}_{7}: \mathrm{ZnSO}_{4}+\mathrm{FeSO}_{4}$ (each @ $2 \mathrm{~g} / \mathrm{kg}$ of seed), $\mathrm{T}_{8}: \mathrm{ZnSO} \mathrm{Zn}_{4}+$ $\mathrm{FeSO}_{4}$ (each @ 4g/kg of seed), $\mathrm{T}_{9}: \mathrm{ZnSO}_{4}+\mathrm{FeSO}_{4}+$ Boron (each @ $2 \mathrm{~g} / \mathrm{kg}$ of seed), $\mathrm{T}_{10}: \mathrm{ZnSO}_{4}+\mathrm{FeSO}_{4}+$ Boron (each @ 4g/kg of seed) , $\mathrm{T}_{11}$ : Only polymer

Genotypes (G): G 1 : Fe \& Zn 2019-2R, G G $_{2}$ Fe \& Zn 2019-4R, G $:$ Fe \& Zn 2019-6R, G 4 : Fe \& Zn 2019-7R, G $:$ Fe \& Zn 2019-10R, G $:$ Fe \& Zn 2019-12R, $\mathrm{G}_{7}: \mathrm{Fe} \& \mathrm{Zn} 2019-16 \mathrm{R}, \mathrm{G}_{8}: \mathrm{Fe} \& \mathrm{Zn} 2019-21 \mathrm{R}, \mathrm{G}_{9}: \mathrm{Fe} \& \mathrm{Zn}$ 2019-22R, $\mathrm{G}_{10}:$ Fe \& Zn 2019-26R, G ${ }_{11}$ : DHANASHAKTHI, G 12 : ICTP 8203, G 13 : VPMH-7 
Table.4 Effect of seed polymerization with micronutrients on seedling dry weight ( $\mathrm{g}$ ) of resultant seeds of biofortified genotypes of pearl millet

\begin{tabular}{|c|c|c|c|c|c|c|c|c|c|c|c|c|c|}
\hline G/T & $T_{0}$ & $\mathbf{T}_{1}$ & $T_{2}$ & $\mathbf{T}_{3}$ & $\mathbf{T}_{4}$ & $\mathbf{T}_{5}$ & $T_{6}$ & $\mathbf{T}_{7}$ & $\mathbf{T}_{8}$ & $\mathbf{T}_{9}$ & $\mathbf{T}_{10}$ & $\mathbf{T}_{11}$ & Mean \\
\hline $\mathbf{G}_{1}$ & 67.03 & 69.93 & 72.87 & 69.67 & 70.27 & 68.51 & 70.23 & 81.03 & 77.67 & 84.27 & 80.45 & 68.9 & 73.4 \\
\hline $\mathbf{G}_{2}$ & 72.12 & 66.3 & 74.67 & 69.86 & 71.04 & 73.17 & 71.32 & 69.62 & 72.36 & 69.77 & 72.36 & 68.21 & 70.9 \\
\hline $\mathbf{G}_{3}$ & 69.07 & 72.81 & 69.08 & 70.67 & 74.56 & 67.03 & 70.27 & 71.27 & 73.17 & 83.2 & 76.95 & 65.37 & 71.95 \\
\hline $\mathbf{G}_{4}$ & 66.06 & 71.64 & 73.27 & 67.59 & 69.87 & 70.32 & 69.26 & 82.18 & 75.97 & 82.95 & 81.95 & 67.87 & 73.24 \\
\hline $\mathbf{G}_{5}$ & 67.97 & 68.56 & 75.69 & 72.75 & 74.17 & 73.43 & 71.17 & 82.77 & 81.12 & 83.3 & 80.07 & 80.31 & 75.94 \\
\hline $\mathbf{G}_{6}$ & 65.47 & 71.88 & 71.67 & 72.97 & 70.62 & 71.17 & 71.67 & 73.66 & 75.98 & 82.67 & 76.15 & 69.77 & 72.81 \\
\hline $\mathbf{G}_{7}$ & 81.42 & 82.62 & 82.87 & 77.57 & 71.36 & 83.2 & 80.62 & 81.36 & 82.32 & 84.23 & 83.4 & 68.87 & 79.99 \\
\hline $\mathbf{G}_{8}$ & 68.32 & 72.97 & 75.41 & 71.05 & 75.97 & 73.99 & 73.52 & 80.38 & 80.29 & 82.21 & 82.01 & 70.23 & 75.53 \\
\hline $\mathbf{G}_{9}$ & 67.67 & 69.57 & 68.52 & 68.87 & 67.97 & 67.77 & 69.87 & 82.28 & 77.27 & 80.73 & 82.95 & 66.67 & 72.51 \\
\hline $\mathbf{G}_{10}$ & 68.67 & 77.57 & 75.52 & 82.87 & 83.26 & 80.77 & 83.39 & 83.28 & 83.27 & 84.83 & 83.5 & 76.67 & 80.3 \\
\hline $\mathbf{G}_{11}$ & 73.67 & 74.88 & 72.42 & 71.47 & 78.67 & 68.52 & 82.87 & 81.59 & 82.18 & 83.21 & 83.3 & 77.87 & 77.55 \\
\hline $\mathbf{G}_{12}$ & 78.67 & 76.57 & 80.52 & 72.87 & 82.36 & 79.77 & 79.87 & 81.26 & 79.63 & 82.32 & 78.63 & 73.67 & 78.85 \\
\hline $\mathbf{G}_{13}$ & 75.32 & 75.93 & 83.4 & 81.67 & 82.32 & 72.51 & 83.23 & 83.03 & 83.21 & 85.89 & 83.84 & 83.09 & 81.12 \\
\hline Mean & 70.88 & 73.17 & 75.07 & 73.07 & 74.8 & 73.09 & 75.18 & 79.52 & 78.8 & 82.28 & 80.43 & 72.12 & \\
\hline $\begin{array}{l}\text { For } \\
\text { comparing } \\
\text { the means of }\end{array}$ & $\begin{array}{c}\text { S.Em } \\
\pm\end{array}$ & $\begin{array}{c}\text { CD at } 1 \\
\%\end{array}$ & & & & & & & & & & & \\
\hline $\mathbf{T}$ & 0.75 & 2.74 & & & & & & & & & & & \\
\hline $\mathbf{G}$ & 0.78 & 2.85 & & & & & & & & & & & \\
\hline T X G & 2.69 & NS & & & & & & & & & & & \\
\hline
\end{tabular}

Note: NS-Non significant

LEGEND: Treatments (T) $\mathrm{T}_{0}:$ Control, $\mathrm{T}_{1}: \mathrm{ZnSO}_{4} @ 2 \mathrm{~g}$ per kg of seed, $\mathrm{T}_{2}: \mathrm{ZnSO}_{4} @ 4 \mathrm{~g}$ per kg of seed, $\mathrm{T}_{3}: \mathrm{FeSO}_{4} @ 2 \mathrm{~g}$ per kg of seed, $\mathrm{T}_{4}: \mathrm{FeSO}_{4} @ 4 \mathrm{~g}$ per kg of seed, $\mathrm{T}_{5}$ : Boron @ $2 \mathrm{~g}$ per kg of seed, $\mathrm{T}_{6}$ : Boron @ 4g per kg of seed, $\mathrm{T}_{7}: \mathrm{ZnSO}_{4}+\mathrm{FeSO}_{4}\left(\mathrm{each} @ 2 \mathrm{~g} / \mathrm{kg}\right.$ of seed), $\mathrm{T}_{8}: \mathrm{ZnSO} 4+\mathrm{FeSO}{ }_{4}(\mathrm{each} @$ $4 \mathrm{~g} / \mathrm{kg}$ of seed), $\mathrm{T}_{9}: \mathrm{ZnSO}_{4}+\mathrm{FeSO}_{4}+$ Boron (each @ $2 \mathrm{~g} / \mathrm{kg}$ of seed), $\mathrm{T}_{10}: \mathrm{ZnSO}_{4}+\mathrm{FeSO}_{4}+$ Boron (each @ 4g/kg of seed), $\mathrm{T}_{11}:$ Only polymer

Genotypes (G): G 1 : Fe \& Zn 2019-2R, G 2 : Fe \& Zn 2019-4R, G 3 : Fe \& Zn 2019-6R, G 4 : Fe \& Zn 2019-7R, G $:$ Fe \& Zn 2019-10R, G 6 : Fe \& Zn 2019-12R, G 7 : Fe \& Zn 2019-16R, G $:$ Fe \& Zn 2019-21R, G $:$ Fe \& Zn 2019-22R, G 10 : Fe \& Zn 2019-26R, G ${ }_{11}$ : DHANASHAKTHI, G 12 : ICTP 8203, G 13 : VPMH-7 
Table.5 Effect of seed polymerization with micronutrients on seedling vigour index-I of resultant seeds of biofortified genotypes of pearl millet

\begin{tabular}{|c|c|c|c|c|c|c|c|c|c|c|c|c|c|}
\hline G/T & $\mathbf{T}_{0}$ & $\mathbf{T}_{1}$ & $\mathbf{T}_{2}$ & $\mathbf{T}_{3}$ & $\mathbf{T}_{4}$ & $\mathbf{T}_{5}$ & $T_{6}$ & $\mathbf{T}_{7}$ & $\mathbf{T}_{8}$ & $\mathbf{T}_{\mathbf{9}}$ & $\mathbf{T}_{10}$ & $\mathbf{T}_{11}$ & Mean \\
\hline $\mathbf{G}_{\mathbf{1}}$ & 2286 & 2750 & 2811 & 2218 & 2482 & 2492 & 2902 & 3140 & 3131 & 3420 & 3127 & 2387 & 2762 \\
\hline $\mathbf{G}_{2}$ & 2070 & 2339 & 2892 & 2378 & 2759 & 2262 & 2245 & 2995 & 2942 & 2619 & 3152 & 2328 & 2582 \\
\hline $\mathbf{G}_{3}$ & 2304 & 2890 & 2475 & 2822 & 2772 & 2496 & 2995 & 3120 & 3041 & 3411 & 3344 & 2328 & 2833 \\
\hline $\mathbf{G}_{4}$ & 2645 & 2710 & 2612 & 2423 & 2786 & 2558 & 3213 & 2954 & 2867 & 3530 & 3159 & 2277 & 2811 \\
\hline $\mathbf{G}_{5}$ & 2458 & 2925 & 2483 & 2431 & 2975 & 3196 & 2781 & 2936 & 2907 & 3500 & 3244 & 2465 & 2858 \\
\hline $\mathbf{G}_{6}$ & 2315 & 2539 & 2659 & 2731 & 2705 & 2589 & 3109 & 3208 & 2738 & 3511 & 3075 & 2236 & 2784 \\
\hline $\mathbf{G}_{7}$ & 2593 & 2867 & 3304 & 3014 & 3207 & 3155 & 3280 & 3521 & 3527 & 3710 & 3170 & 3256 & 3217 \\
\hline $\mathbf{G}_{8}$ & 2607 & 2427 & 2939 & 2885 & 2934 & 2176 & 2585 & 3391 & 2703 & 3338 & 3164 & 2537 & 2807 \\
\hline $\mathbf{G}_{9}$ & 2448 & 2669 & 2791 & 2917 & 2924 & 2303 & 2415 & 3126 & 2874 & 3409 & 3378 & 2815 & 2839 \\
\hline $\mathbf{G}_{10}$ & 2561 & 3108 & 3532 & 3482 & 3121 & 3442 & 3306 & 3490 & 3447 & 3823 & 3610 & 3079 & 3333 \\
\hline $\mathbf{G}_{11}$ & 2538 & 2801 & 3270 & 2985 & 3176 & 2424 & 2965 & 2931 & 3268 & 3148 & 3379 & 2995 & 2990 \\
\hline $\mathbf{G}_{12}$ & 3113 & 2783 & 3187 & 2652 & 2797 & 3201 & 2815 & 3254 & 3389 & 3548 & 3243 & 3003 & 3082 \\
\hline $\mathbf{G}_{13}$ & 2995 & 3184 & 3472 & 3473 & 3509 & 3538 & 3331 & 3526 & 3518 & 3908 & 3653 & 3140 & 3437 \\
\hline $\begin{array}{c}\text { Mean } \\
\text { For } \\
\text { comparing the } \\
\text { means of }\end{array}$ & $\begin{array}{c}2533 \\
\text { S.Em } \pm\end{array}$ & $\begin{array}{c}2769 \\
\text { CD at } 1 \\
\%\end{array}$ & 2956 & 2801 & 2935 & 2756 & 2919 & 3200 & 3104 & 3452 & 3284 & 2680 & \\
\hline $\mathbf{T}$ & 0.75 & 2.74 & & & & & & & & & & & \\
\hline G & 0.78 & 2.85 & & & & & & & & & & & \\
\hline T X G & 2.69 & NS & & & & & & & & & & & \\
\hline
\end{tabular}

Note: NS-Non significant

LEGEND: Treatments (T) $\mathrm{T}_{0}$ : Control, $\mathrm{T}_{1}: \mathrm{ZnSO}_{4} @ 2 \mathrm{~g}$ per kg of seed, $\mathrm{T}_{2}: \mathrm{ZnSO}_{4} @ 4 \mathrm{~g}$ per kg of seed, $\mathrm{T}_{3}: \mathrm{FeSO}_{4} @ 2 \mathrm{~g} \mathrm{per} \mathrm{kg} \mathrm{of} \mathrm{seed,} \mathrm{T}_{4}: \mathrm{FeSO}_{4} @ 4 \mathrm{~g}$ per kg of seed, $\mathrm{T}_{5}$ : Boron @ $2 \mathrm{~g}$ per kg of seed, $\mathrm{T}_{6}$ : Boron @ $4 \mathrm{~g}$ per kg of seed, $\mathrm{T}_{7}: \mathrm{ZnSO}_{4}+\mathrm{FeSO}_{4}\left(\mathrm{each} @ 2 \mathrm{~g} / \mathrm{kg}\right.$ of seed), $\mathrm{T}_{8}: \mathrm{ZnSO}_{4}+\mathrm{FeSO}_{4}($ each @ $9 \mathrm{~g}$ $/ \mathrm{kg}$ of seed), $\mathrm{T}_{9}: \mathrm{ZnSO}_{4}+\mathrm{FeSO}_{4}+$ Boron (each @ 2g/kg of seed), $\mathrm{T}_{10}: \mathrm{ZnSO}_{4}+\mathrm{FeSO}_{4}+$ Boron (each @ 4g/kg of seed), $\mathrm{T}_{11}:$ Only polymer

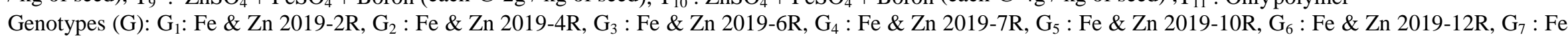

\& Zn 2019-16R, G $:$ Fe \& Zn 2019-21R, G $:$ Fe \& Zn 2019-22R, G 10 : Fe \& Zn 2019-26R, G 11 : DHANASHAKTHI, G 12 : ICTP 8203, G 13 : VPMH-7 
Table.6 Effect of seed polymerization with micronutrients on seedling vigour index-II of resultant seeds of biofortified genotypes of pearl millet

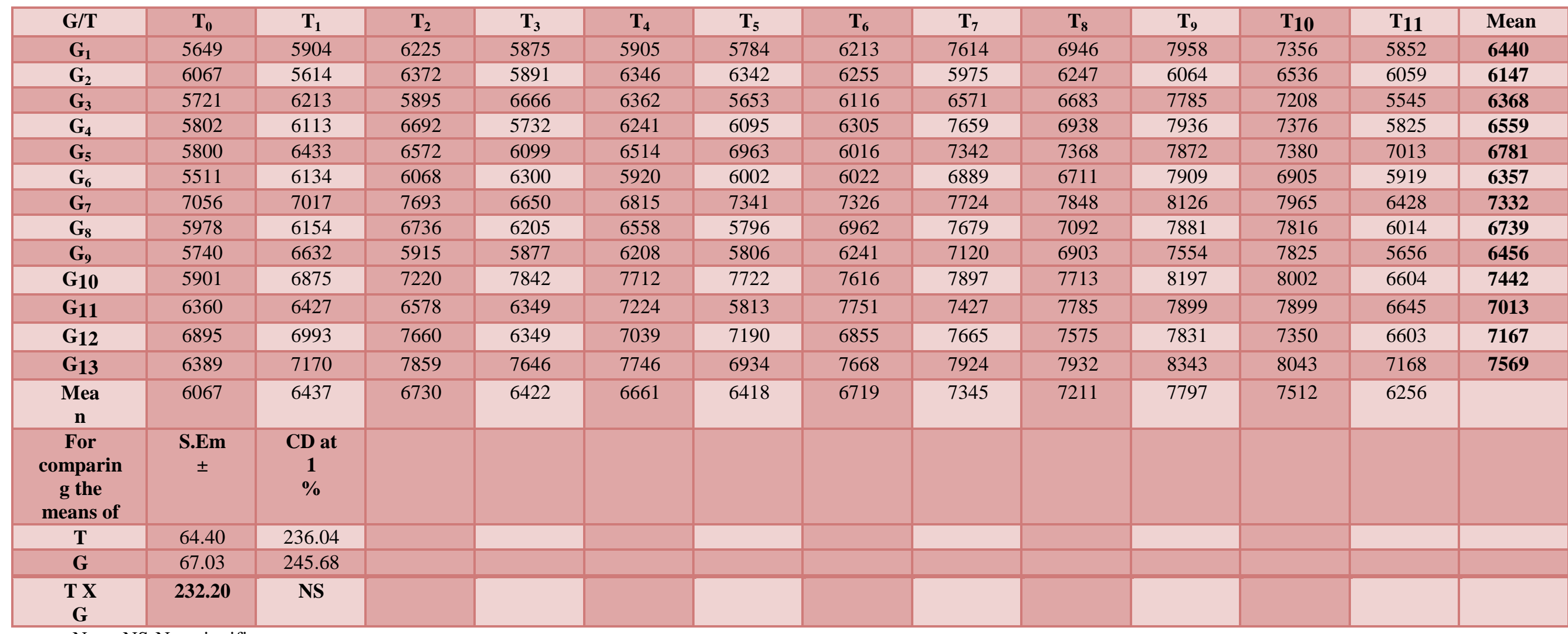

Note: NS-Non significant

LEGEND: Treatments (T) $\mathrm{T}_{0}:$ Control, $\mathrm{T}_{1}: \mathrm{ZnSO}_{4} @ 2 \mathrm{~g}$ per kg of seed, $\mathrm{T}_{2}: \mathrm{ZnSO}_{4} @ 4 \mathrm{~g}$ per kg of seed, $\mathrm{T}_{3}: \mathrm{FeSO}_{4} @ 2 \mathrm{~g}$ per kg of seed, $\mathrm{T}_{4}: \mathrm{FeSO} \mathrm{S}_{4} @ 4 \mathrm{~g}$ per kg of seed, $\mathrm{T}_{5}$ : Boron @ 2g per kg of seed, $\mathrm{T}_{6}:$ Boron @ $4 \mathrm{~g}$ per kg of seed, $\mathrm{T}_{7}: \mathrm{ZnSO}_{4}+\mathrm{FeSO}_{4}$ (each @ 2g/kg of seed), $\mathrm{T}_{8}: \mathrm{ZnSO}_{4}+\mathrm{FeSO}_{4}($ each @ $4 \mathrm{~g} / \mathrm{kg}$ of seed), $\mathrm{T}_{9}: \mathrm{ZnSO}_{4}+\mathrm{FeSO}_{4}+$ Boron (each @ $2 \mathrm{~g} / \mathrm{kg}$ of seed), $\mathrm{T}_{10}: \mathrm{ZnSO}_{4}+\mathrm{FeSO}_{4}+$ Boron (each @ 4g/kg of seed), $\mathrm{T}_{11}:$ Only polymer

Genotypes (G): G $:$ Fe \& Zn 2019-2R, G 2 : Fe \& Zn 2019-4R, G $:$ Fe \& Zn 2019-6R, G G $_{4}:$ Fe \& Zn 2019-7R, G $:$ Fe \& Zn 2019-10R, G $:$ Fe \& Zn 2019-12R, G $:$ Fe

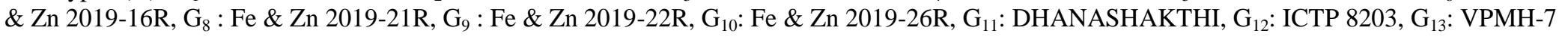


The increased shoot and root length might be due to seeds with higher test weight shall have higher germination potential and seedling growth, this intern might have increased the metabolic activity of indole acetic acid and auxin (Basaria Begam and Krishnasamy, (2003), the increased seedling vigour index might be due to higher root and shoot length and higher germination percentage. Similar results were reported by Harish Babu et al., (2005), Sherin Susan et al., (2005), Vinodkumar et al., (2013), Handiganoor et al., (2018).

The seed yield varied significantly among the different biofortified genotypes of pearlmillet, The increased seed yield was recorded in $\mathrm{G}_{13}$ [VPMH-7] (2667 kg/ha) fallowed by $\mathrm{G}_{10}[\mathrm{Fe} \& \mathrm{Zn}$ 2019-26R] (2609 kg/ha) and $\mathrm{G}_{7}[\mathrm{Fe} \& \mathrm{Zn} 2019-16 \mathrm{R}]$ (2517 kg/ha) While, the seed yield recorded in $\mathrm{G}_{2}[\mathrm{Fe} \& \mathrm{Zn} 2019$ 4R] (1933 kg/ha).

The seed quality parameters varied significantly among different biofortified genotypes of pearlmillet, The increased seedling vigour index-I (3437) and seedling vigour index-II (7568) observed in $\mathrm{G}_{13}$ [VPMH-7]. The increased seedling vigour index-I and seedling vigour index-II mainly attributed higher seed germination percentage, seedling legth and Seedling dry weight were recorded in $\mathrm{G}_{13}$ [VPMH-7] (93.26, $37.14 \mathrm{~cm}$ and $81.12 \mathrm{~g}$ respectively) fallowed by $\mathrm{G}_{10}[\mathrm{Fe}$ \& Zn 2019-26R] (92.53, $35.92 \mathrm{~cm}$ and 80.30 g respectively) and $\mathrm{G}_{7}$ [Fe \& $\mathrm{Zn}$ 2019-16R] (91.69, $35.29 \mathrm{~cm}$ and $79.99 \mathrm{~g}$ respectively) While, the lower germination percentage, shoot length, root length and seedling dry weight recorded in $\mathrm{G}_{2}[\mathrm{Fe} \& \mathrm{Zn}$ 2019-4R] (86.70, $31.64 \mathrm{~cm}$ and $70.99 \mathrm{~g}$ respectively).

The seed yield per hectare was not significantly influenced by the seed polymerization with micronutrients in bio fortified genotypes of pearl millet. The higher seed yield per hactare of 27.54 percent over control. The maximum seed yield (2855 $\mathrm{kg} / \mathrm{ha}$ ) was recorded in $\mathrm{G}_{13}$ [VPMH-7] and seed polymerization with $\mathrm{ZnSO}_{4}+\mathrm{FeSO}_{4}+$ boron@ $2 \mathrm{~g}$ per kg of seed followed by $\mathrm{G}_{10}$ [Fe \& Zn 2019-26R] seed polymerization with $\mathrm{ZnSO}_{4}+\mathrm{FeSO}_{4}+$ boron @ 2 g per kg of seed $(2819 \mathrm{~kg} / \mathrm{ha})$ and the seeds of $\mathrm{G}_{7}[\mathrm{Fe} \&$ $\mathrm{Zn}$ 2019-16R] seed polymerization with $\mathrm{ZnSO}_{4}+\mathrm{FeSO}_{4}+$ boron @ $2 \mathrm{~g}$ per kg of seed $(2813 \mathrm{~kg} / \mathrm{ha})$ While, the minimum seed yield was noticed in $\mathrm{G}_{2}$ [Fe \& $\mathrm{Zn} 2019-4 \mathrm{R}$ ] control without seed polymerization (1668 kg/ha) The increase in seed yield as a result of seed polymerization with micronutrients might be attributed to the role played by micronutrients in activating dehydrogenase and other enzymes. These micronutrients are also necessary for the biosynthesis of IAA, the growth regulator, which is essential for normal enlargement of cells. It is also a constituent of amino acids, from which protein and enzymes are synthesized. Similar results obtained by Shakuntala et al., (2012) in sunflower, Shinde et al., (2018) in pigeon pea and Handiganoor et al., (2018) in pigeon pea.

Seed quality parameters did not differed significantly due to interaction of seed polymerization with a micronutrients with different bio-fortified genotypes of pearl millet. The increased seedling vigour index-I (3907) and seedling vigour index-II (8342) was observed in $\mathrm{G}_{13}$ [VPMH-7] and seed polymerized with $\mathrm{ZnSO}_{4}+\mathrm{FeSO}_{4}+$ boron each @ $2 \mathrm{~g}$ per $\mathrm{kg}$ of seed. However numerically higher seedling vigour index-I and seedling vigour index-II was mainly attributed to higher germination percentage, seedling length and seedling dry weight were recorded in $\mathrm{G}_{13}$ [VPMH-7] seed polymerized with $\mathrm{ZnSO}_{4}+\mathrm{FeSO}_{4}+$ boron each @ 2g per $\mathrm{kg}$ of seeds $(97.13 \%, 39.20 \mathrm{~cm}$ and $85.99 \mathrm{~g}$ respectively) followed by $\mathrm{G}_{10}$ [Fe \& $\mathrm{Zn} 2019$ 26R] seed polymerized with $\mathrm{ZnSO}_{4}+\mathrm{FeSO}_{4}$ 
+ boron each @ 2g per kg of seeds, (96.63\%, $38.20 \mathrm{~cm}$ and $84.83 \mathrm{~g}$ respectively), G7 [Fe \& $\mathrm{Zn} 2019-16 \mathrm{R}]$ seed polymerized with $\mathrm{ZnSO}_{4}$ $+\mathrm{FeSO}_{4}+$ boron each @ $2 \mathrm{~g}$ per kg of seeds ( $96.47 \%, 38.38 \mathrm{~cm}$ and $84.23 \mathrm{~g}$ respectively), While, the lower seed quality parameters was seen in $\mathrm{G}_{2}$ [Fe \& $\mathrm{Zn} 2019-4 \mathrm{R}$ ] control with no seed polymerization $(84.13 \%, 28.10 \mathrm{~cm}$ and $72.12 \mathrm{~g}$ respectively). This is having positive relation to seed development which is reflected through the size and test weight of seed and the micronutrients present in it. This is in turn dependent on efficient synthesis, accumulation of food metabolites such as protein, carbohydrates and their translocation from source to the developing seed at greater ease, The increased shoot and root length might be due to seeds with higher test weight shall have higher germination potential and seedling growth, this intern might have increased the metabolic activity of indole acetic acid and auxin (Basaria Begam and Krishnasamy, 2003) through micronutrients and its translocation leading to early germination, cell division and elongation leading to increase in root and shoot length. Similar results were reported by Srimathi et al., (2007), Shinde et al., (2017).

The increase in seedling dry weight was due to better seedling length and also greater vigour reflected in early stage and higher percentage of germination of seeds that had reached autotrophic stage well in advance than others. Increase in dry weight might further be enhanced due to enhanced lipid utilisation through glyoxalate cycle, a primitive metabolic pathway thereby, facilitating the conversion of acetate into nucleic acid (Sherin Susan et al., 2005), Handiganoor et al., (2018), so As a result of improved germination percentage, seedling length and seedling dry weight due to imposed treatments the seedling vigour index I and II differed significantly.
It could be concluded that seed polymerization $(5 \mathrm{ml} / \mathrm{kg}$ of seed $)$ of pearlmillet seeds coupled with micronutrients viz., $\mathrm{ZnSO} 4+\mathrm{FeSO} 4+$ Boron each at $2 \mathrm{~g}$ per $\mathrm{kg}$ of seed found to be better treatment to record higher seed yield and quality parameters in pearlmillet.

\section{References}

Abdul-Baki, A. A. and Anderson, J. D., 1973, Vigour determination in soybean seeds by multiple criteria. Crop Sci., 13: 630633.

Anonymous. Guidelines for use of micronutrients, soil ameliorants and integrated nutrient management practices in National Food Security Mission States, 2011, 1-27.

Anonymous. www.icmr.nic.in, 2014.

Anonymous, 2020, Area production productivity of millets, Ministry of Agriculture and Farmers welfare, Directorate of Economics and Statistics, Govt. of India. (ON1151).

Basaria Begum, J. and Krishnasamy, V., 2003, Effect of seed hardening and pelleting on seed germination and vigour in black gram. Seed Res., 3(2): 194-199.

Bharathi A, Srinivasan J. Seed quality enhancement- Principles and practices. Published by scientific publishers (India), 2010, 234-235.

Dell B, Huang L. Physiological responses of plants to boron. Plant Soil. 1997; 193:103-120.

Evans LE, Bhatt GW. A non-destructive technique for measuring seedling vigour in wheat. Canadian J Plant Sci. 1977; 57:983-985.

Gomez KA, Gomez AA. Statistical procedures for agricultural research, 2ndedition, A Willey International Science Publication, New York (USA), 2010, 20-29. 
Handiganoor, M. G., Patil, S. B. and Vasudevan, S. N., 2018. effect of Seed coating polymer and micronutrients on stomatal conductance and resistance at different growth stages of pigeonpea. Advances in Research, 1-8.

Harish Babu, B. N., Kempegowda, M. L., Kalappa, V. P. and Rudra Naik, V, 2005, Comparative evaluation of different pelleting materials on seed performance of French bean and green gram. Karnataka J. Agric. Sci., 18(1): 32-35.

Hirschi, K. D. (2009). Nutrient biofortification of food crops. Ann. Rev. Nutr. 29, 401-421. doi: 10.1146/annurev-nutr-080508-141143

Johnson SE, Lauren JG, Welch RM, Duxbury JM. A comparison of the effects of micronutrient seed priming and soil fertilization on the mineral nutrition of chickpea (Cicer arietinum), lentil (Lens culinaris), rice (Oryza sativa) and wheat (Triticum aestivum) in Nepal. Exper. Agric. 2005; 41:427-448.

Kaleeswari RK, Rajeswari R. Prabhaharan. J. A handbook of soil fertility. Satish serial publishing house, 2013

Shakuntala, N. M., Vasudevan, S. N., Shankergoud, I. and Rakesh Mathad, 2012, Polymer film coating for precision seeding in sunflower. AgroInformatics and Precision Agric., pp.339-340
Sharma, J., Dhiman, K. C., Sharma, J. K. and Kumar, R., 2017. Effect of Seed Coating on Seed Yield and Related Parameters in Quality Protein Maize Hybrid Hqpm 1 (Zea mays L.). Himachal J. Agric. Res., 43(1):68-72.

Singh B, Natesan SKA, Singh BK, Usha K. Improving zinc efficiency of cereals under zinc deficiency. Curr. Sci. 2003; 88:36-14.

Sherin Susan John, Bharati, Natesan, P. and Raja, K., 2005, Seed film coating technology for maximizing the growth and productivity of maize. Karnataka. J. Agric. Sci., 18(2): 349-356.

Shinde, P., Doddagoudar, S. R. and Vasudevan, S. N., 2017. Influence of seed polymer coating with micronutrients and foliar spray on seed yield of chickpea (Cicer arietinum L.). Legume Research-An Inter. J., 40(4), pp.704-709.

Srimathi, P., Kavitha, S. and Renugadevi, J., 2007, Influence of seed hardening and pelleting on seed yield and quality in green gram (Vigna radiate) cv. Co-6. Indian. J. Agric. Res., 41(2): 122-126.

Vinodkumar, B. S., Vyakarnahal, Dhananjaya. P., Yegappa Hipparagi and Asha, A. M., 2013, Effect of seed polymer coating on field performance and quality of pigeonpea (Cajanus cajan (L.) Millsp.). Env. and Ecology., $31(1): 43-46$.

\section{How to cite this article:}

Gireesh, S., S. B. Patil, Ashok S. Sajjan and Patil, M. D. 2021. Effect of Seed Polymerization with Micronutrients on Seed Yield and Quality in Biofortified Genotypes of Pearl Millet [Pennisetum glaucum (L.)]. Int.J.Curr.Microbiol.App.Sci. 10(02): 46-58. doi: https://doi.org/10.20546/ijcmas.2021.1002.006 